\title{
Effect of Off-Body Laser Discharge on Drag Reduction of Hemisphere Cylinder in Supersonic Flow
}

\author{
Nadia Kianvashrad* and Doyle Knight ${ }^{\dagger}$ \\ Rutgers - The State University of New Jersey, New Brunswick, New Jersey 08903, USA \\ Stephen P. Wilkinson, ${ }^{\ddagger}$ Amanda Chou, ${ }^{\ddagger}$ Robert A. Horne, ${ }^{\S}$ \\ Gregory C. Herring, "George B. Beeler, ${ }^{\ddagger}$ and Moazzam Jangdall \\ NASA Langley Research Center, Hampton, VA, 23681, USA
}

\begin{abstract}
The interaction of an off-body laser discharge with a hemisphere cylinder in supersonic flow is investigated. The objectives are 1) experimental determination of the drag reduction and energetic efficiency of the laser discharge, and 2) assessment of the capability for accurate simulation of the interaction. The combined computational and experimental study comprises two phases. In the first phase, laser discharge in quiescent air was examined. The temporal behavior of the shock wave formed by the laser discharge was compared between experiment and simulation and good agreement is observed. In the second phase, the interaction of the laser discharge with a hemisphere cylinder was investigated numerically. Details of the pressure drag reduction and the physics of the interaction of the heated region with the bow shock are included. The drag reduction due to this interaction persisted for about five characteristic times where one characteristic time represents the time for the flow to move a distance equal to the hemisphere radius. The energetic efficiency of laser discharge for the case with $50 \mathrm{~mJ}$ energy absorbed by the gas is calculated as 3.22.
\end{abstract}

\section{Introduction}

SEveral experimental and computational studies have demonstrated drag reduction in supersonic flow $S$ due to energy deposition. Artem'ev et al. ${ }^{1}$ showed experimentally and computationally that energy deposition by a thin heated filament could reduce drag up to $80 \%$ over a blunt cylinder at Mach 2.0 and 3.1. Tretyakov et al. ${ }^{2}$ observed experimentally that high frequency laser discharge pulses could reduce drag over conical and hemispherical objects up to $45 \%$ at Mach 2.0. Riggins et al. ${ }^{3}$ showed computationally that laser discharge reduced drag by $30 \%$ to $50 \%$ for blunt bodies in hypersonic flows at Mach 6.5 and 10 . Lashkov et al. ${ }^{4}$ showed experimentally that microwave discharge reduces the centerline pressure of a blunt cylinder and hemisphere at Mach 2.1. Adelgren et al. ${ }^{5}$ showed experimentally that laser discharge reduces centerline pressure of a hemisphere by $40 \%$ at Mach 3.45 . Knight et al. ${ }^{6}$ reported that both experiment and computation show reduction in centerline pressure over a hemisphere due to microwave discharge at Mach 2.1. Schülein et al. ${ }^{7}$ observed that single and double pulsed laser discharge reduced centerline pressure on a hemisphere at Mach 2.0. Kim et al. ${ }^{8}$ experimentally reported drag reduction up to $21 \%$ and energetic efficiency up to $500 \%$ for repetitive laser discharge up to $80 \mathrm{kHz}$ in front of a right circular cylinder. Kremeyer et al. ${ }^{9}$ indicated a drag reduction up to $96 \%$ with an energetic efficiency of up to $6500 \%$ for line source

${ }^{*} \mathrm{PhD}$ Candidate, Department of Mechanical and Aerospace Engineering, AIAA Student Member. Email: nadiakianvashrad@gmail.com

${ }^{\dagger}$ Professor, Department of Mechanical and Aerospace Engineering, AIAA Member. Email: doyleknight@gmx.com

${ }^{\ddagger}$ Research Aerospace Engineer, Flow Physics \& Control Branch, AIAA Senior Member.

$\S$ Research Electronics Engineer, Revolutionary Aviation Technologies Branch.

IResearch Physicist, Advanced Measurements \& Data Systems Branch.

"Research Aerospace Engineer, Flow Physics \& Control Branch.

This work is declared a work of the U.S. Government and is not subject to copyright protection in the United States. 
deposition based on the prior work of Plooster. ${ }^{10,11}$ A recent review of computational and experimental studies of drag reduction by energy deposition is presented in Knight. ${ }^{12}$

It is clear that a wide variation in energy deposition performance has been reported within the parameter space of deposition geometry, energy input level, and frequency. To help provide a basis for sorting out the many reported results, we examine, both computationally and experimentally, the canonical case of a single, laser-induced, point discharge upstream of a supersonic blunt body. This differs from previous studies where high repetition rates were used to produce a continuous effect. Also, this study involves only laser-induced discharges and does not address alternative methods such as electrode discharges or electrodeless microwaves. The objective of this work is twofold: experimental evaluation of the gas dynamic energetic efficiency, defined as the ratio of the energy saved (due to the drag reduction) to the energy actually absorbed by the gas, and assessment of the capability of CFD prediction of drag reduction. The research is conducted in two phases, namely, 1) laser discharge in quiescent air, and 2) laser discharge and its interaction with a hemisphere cylinder at Mach 2.

\section{Description of Experiments}

The experiments are performed at the NASA Langley Research Center. The first phase is a laser discharge in quiescent air at different ambient pressures. The second phase is laser discharge in front of a hemisphere cylinder at Mach 2 and its interaction with the body. Results for the first phase are presented in section IV.B. The second phase is in progress.

\section{A. Laser Discharge in Quiescent Air}

Benchtop experiments were performed to provide validation for the CFD code and to mitigate risk prior to the wind tunnel experiments. These measurements were used to determine the evolution of the shock radius over time for different absorbed laser energies. The benchtop experiments also served to address concerns about achieving breakdown in low-density environments similar to the NASA LaRC 20-inch Supersonic Wind Tunnel. The goal of these benchtop experiments was to reduce the risk of burning the 3-inch thick wind tunnel windows while repeatably achieving laser-induced breakdown at low densities. Variations in energy lost through the breakdown, location of breakdown, and the consistency of the breakdown were monitored for different iterations of focusing optics.

A schematic of the setup for the benchtop experiment is presented in Fig. 1. On the bench, an Nd:YAG laser beam was directed into a test cell, which could be set to a desired pressure within an uncertainty of $\pm 1 \%$. The Nd:YAG laser used was capable of producing $10-\mathrm{Hz}$ pulses approximately $10 \mathrm{~ns}$ in duration with a maximum energy of $930 \mathrm{~mJ}$ per pulse. The $532-\mathrm{nm}$ beam diameter was approximately $7 \mathrm{~mm}$. The beam was sampled prior to and after the test cell with two laser energy meters. The energy losses across each optical element in the beam path were carefully recorded and accounted for in the readings of each of the energy meters. Thus, the readout of the energy meters could be used to determine the loss in energy $(\Delta E)$ across the region where laser-induced breakdown occurred. Focusing optics were placed in the laser beam path just before the test cell so that the laser-induced breakdown process was visible in the side windows of the test cell. A beam stop was placed at the end of the beam path for safety.

The schlieren system was set up at an axis perpendicular to the laser beam path. A continuous white LED was used as the light source of the schlieren system and the images were collected with a 12-bit intensified CCD camera. The intensifier of the CCD camera had a higher quantum efficiency in the UV wavelengths. The camera was set to take up to 300 total frames at an exposure of $200 \mathrm{~ns}$ to $1 \mu \mathrm{s}$. A set of frames was taken at a set time interval $(\Delta t)$ after each laser shot was fired and then averaged to improve the signal-tonoise ratio of the schlieren image. Multiple averaged frames were taken at various $\Delta t$ after each laser pulse was fired. The combination of these averaged images presented a time history showing the evolution of the thermal perturbation and shock wave. 


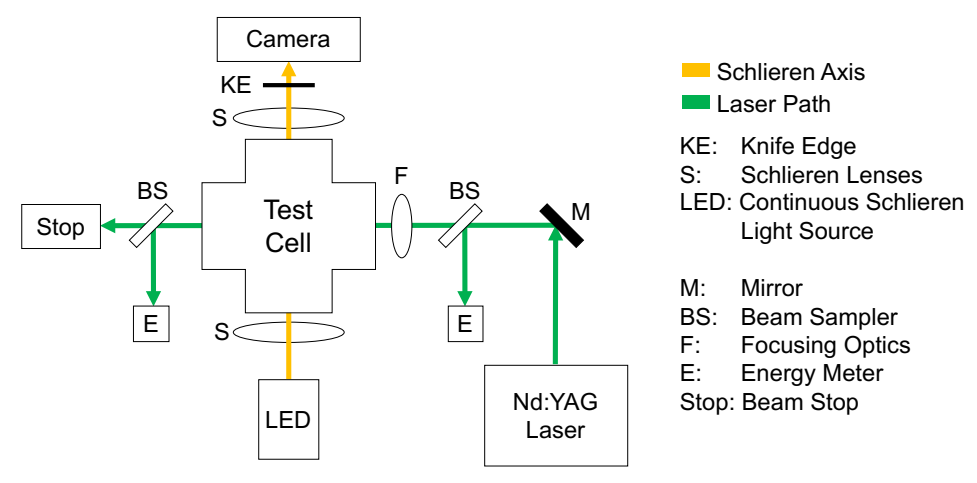

Figure 1. A schematic of the benchtop experiment setup.

\section{B. Laser Discharge in Front of a Hemisphere Cylinder at Mach 2}

A hemisphere cylinder blunt body wind tunnel test is currently being planned for testing at Mach 2 in the NASA Langley 20 Inch Supersonic Wind Tunnel. ${ }^{13}$ The purpose of the test is to measure the model's impulse response due to a single, or a low frequency, noninteracting sequence of, laser energy deposition pulses. This approach is predicated on the assumption that all of the relevant flow physics of the interaction between the energy deposition and the model's dynamic response are contained in a single pulse. The benefit of this approach is that it avoids the need for a high pulse-rate laser with its attendant costs and operational issues. On the other hand, dynamic impulse measurements in a supersonic wind tunnel require technique development and calibration beyond more conventional, steady-state measurements. Foremost among the uncertainties in impulse measurement is the background tunnel structural vibration, and its possible effect on the signal-to-noise ratio of the measurement system.

As illustrated in Fig. 2, the model consists of an aluminum hemisphere, $50.8 \mathrm{~mm}$ in diameter, with a tangent cylinder of equal length. The model is supported by an axial pair of low friction, commercial air bearings, and a hollow, low mass, precision diameter aluminum sting. Efflux air from the air bearing is captured and removed by annular glands built into the strut at both ends of each bearing. The aft end of the sting contacts a fixed-free cantilevered elastic steel flexure beam that is sized for the anticipated mean model drag. The base of the hemisphere-cylinder is shielded with an extension fairing and single-pass labyrinth gland forming a close-clearance, noncontact, annular passage. This feature allows equalization of the base pressure to free-stream static pressure while preventing separated base flow, a requirement for accurate drag measurement. The aft end of the model is housed in an airtight instrumentation enclosure that is vented through an external tube to a free stream static port on the tunnel wall downstream of the model bow shock. A high precision, triangulation-type, laser position sensor with a range of $2 \mathrm{~mm}$ and a resolution of $0.03 \mu \mathrm{m}$ at a maximum frequency of $20 \mathrm{kHz}$ is used to monitor the sting position for both mean and impulse loads. The model sting is outfitted with a miniature, low mass, axial accelerometer as an alternative method of measuring impulse loading. Flush static pressure ports $(d=0.25 \mathrm{~mm})$ starting at the forward stagnation point and proceeding along a hemispherical arc and the cylinder body are also provided to characterize the mean static pressure field. Photographs of the completed model assembly and the empty test section of the wind tunnel are shown in Figs. 3(a) and 3(b). During testing, the hemisphere-cylinder model nose will be positioned on the tunnel centerline within the field of view of the test section windows approximately at the location where the free stream pitot probe is now shown in the photograph.

The object of the experiment is to determine the model impulse caused by a rapidly convecting, low density slug of air from the energy deposition laser pulse, and to compare that to the prediction of the companion CFD portion of this study. The wind tunnel model is a 2nd order, damped, spring-mass system so that the impulse can be readily derived from the sting's position or acceleration time history. In the case where the model strut is completely stationary, the computation is straightforward and examples are shown below. In the current experiment, however, the wind tunnel floor to which the model strut is mounted must be assumed to be moving in some unknown vibration mode. Such motion, in principle, can range from simple, single-axis sinusoidal motion to increasingly complex multiaxis vibration signatures. The tunnel floor 


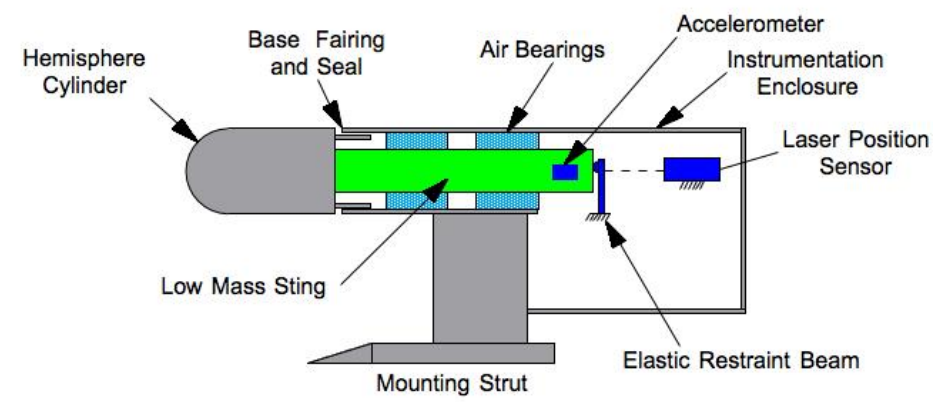

Figure 2. Essential features of the hemisphere-cylinder model and impulse measurement system.

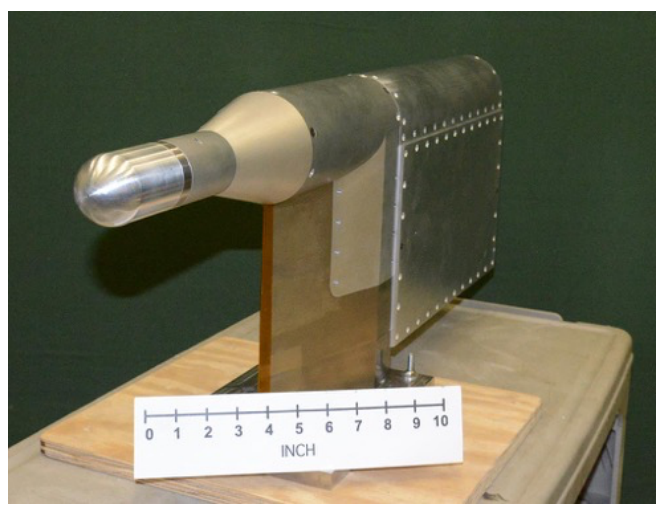

(a) Photograph of completed hemispherecylinder model

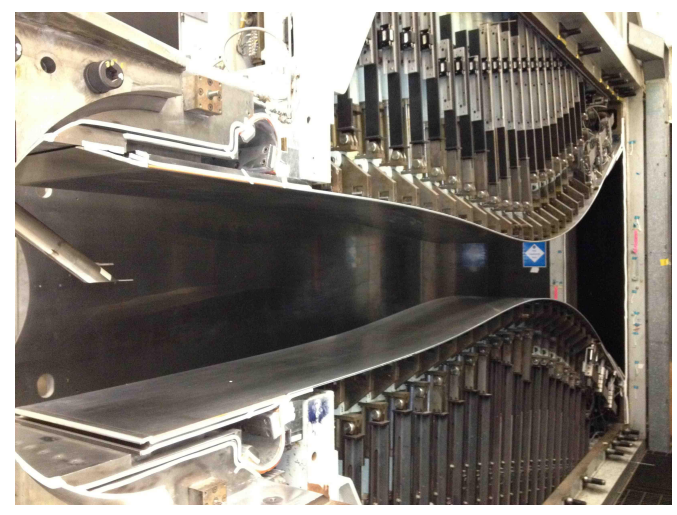

(b) Photograph of the NASA Langley 20 Inch Low Speed Wind Tunnel with the variable Mach number nozzle set at $\mathrm{M}=2$ (Near side walls removed)

Figure 3. Hemisphere cylinder model and wind tunnel cross section.

motion and aerodynamic loading can in turn excite various vibration modes in the model assembly of varying complexity including structural resonances. The primary risk and challenge of this experiment, therefore, is to ascertain under what model vibration conditions, can the input impulse be accurately extracted from dynamic position and/or acceleration sting data. It is assumed that any longitudinal and flexural waves in the sting itself are second order compared to the rigid body motion of the entire model assembly or relative motion between model parts. (Magnetic eddy-current damping may be useful in limiting excessive axial vibration excursions but is not currently installed in the system.) While preparations are being made to conduct actual wind tunnel tests, a brief benchtop exercise was conducted on the model to help characterize its impulse behavior under both stationary and structural vibration conditions.

A vibration motor was rigidly clamped to the model frame and a pendulum impact apparatus was used to generate an impulse magnitude similar to what might be expected from actual laser energy deposition. The pendulum impact method is assumed to be fully elastic, as opposed to a largely dissipative interaction between the heated air slug and the hemispherical model in the wind tunnel test. Nonetheless, the pendulum method allows study of the system's measurement sensitivity and accuracy, in the absence of actual flow tests. The vibration motor was a small DC motor with approximately 3.8 gram weight offset from the shaft by $11.5 \mathrm{~mm}$ and operated at rotational speeds up to $3600 \mathrm{RPM}(f=\omega /(2 \pi)=100 \mathrm{~Hz})$. The pendulum consisted of an $18 \mathrm{~mm}$ hardened steel ball bearing with a mass of $m=23.5 \mathrm{~g}$ suspended from a pair of nylon monofilament lines of negligible mass arranged to strike the sting's hardened steel mounting face at the minimum of the pendulum swing. The effective pendulum length was $675 \mathrm{~mm}$. The mass of the floating sting in the air bearings was $M=1.164 \mathrm{~kg}$ and the pendulum was dropped from a vertical height of $5 \mathrm{~mm}$. 
Assuming a fully elastic collision, the impulse exerted on the sting by the ball bearing can be calculated from simultaneous application of conservation of momentum and kinetic energy ${ }^{14}$ before and after the collision. For the above conditions, $I=0.015$ newton-seconds. By varying the vibration motor rotational speed, various vibration modes and amplitudes could be excited in the air-bearing-floated sting, strut and model frame. Only the axial component of sting displacement and acceleration was measured.

Fig. 4 shows resonant behavior of the sting over a range of input frequencies for both the independently measured displacement and acceleration signals. The resonant peak is located at approximately $f=58.6 \mathrm{~Hz}$, the midpoint of the slightly flat plateau. From this, the effective spring constant can be estimated as $k=M \omega^{2}=157.8 \mathrm{kN} / \mathrm{m}$. The spring constant can also be estimated from the mechanical properties and dimensions of the steel elastic allowing the resonant peak to be moved, if required, by changing the material and mechanical design of the elastic flexure.

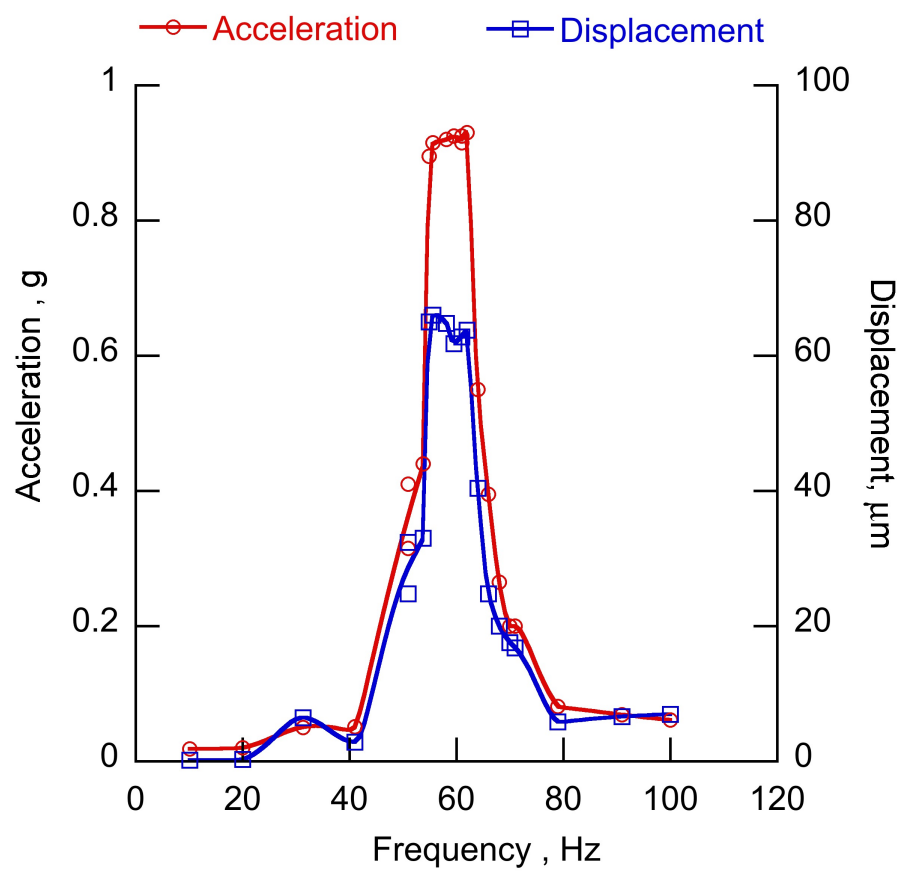

Figure 4. Resonant behavior of model sting in dual air bearing support with elastic restraint.

Figs. 5(a-c) are examples of the pendulum impact effect on sting displacement at three vibration motor speeds, $f=50.3,55.2$, and $100 \mathrm{~Hz}$. In Fig. 5(a) for the relatively benign, low amplitude displacement of \pm 20 microns for the preresonance case of $f=50.3 \mathrm{~Hz}$, clean transitions between the pre- and post-impact signatures are clearly visible and amenable momentum and kinetic energy analysis to extract the impulse. In Fig $5(\mathrm{~b})$ at $f=55.2 \mathrm{~Hz}$, close to the resonant peak at $f=58.6 \mathrm{~Hz}$, with initial amplitudes on the order of \pm 100 microns, the transitions due to a fixed impulse magnitude become increasingly difficult to discern with likely reduced accuracy. At a high frequency, well removed from resonance, the impact transitions also become obscure, in this case not because of high initial amplitudes, but rather due to increased complexity in the vibration signatures. From this exercise, it is clear that a low vibration wind tunnel environment is preferred with measurement accuracy increasingly adversely impacted as vibration amplitude and complexity increase.

As a quantitative example, for the case of no background vibration, the governing equations for a damped, 2nd-order, spring-mass system are:

$$
x(t)=\frac{I}{m \omega_{d}} \exp ^{\left(-\zeta \omega_{n} t\right)} \sin \left(\omega_{d} t+\phi\right)
$$




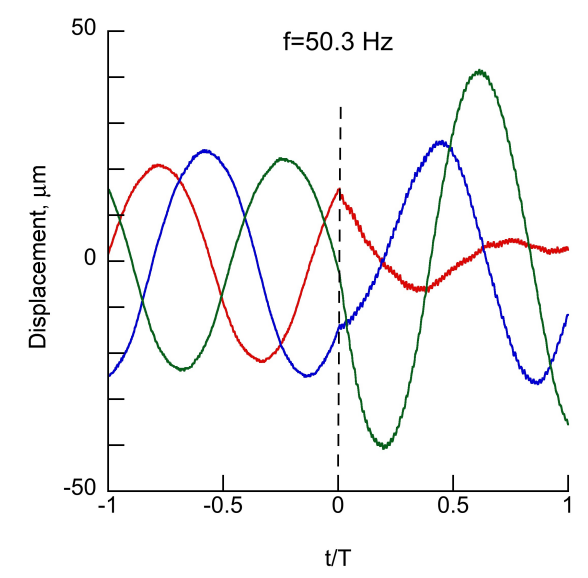

(a) Sting displacement under $I=0.015 \mathrm{~N}$-s impact with vibration motor set at $f=50.3 \mathrm{~Hz}$

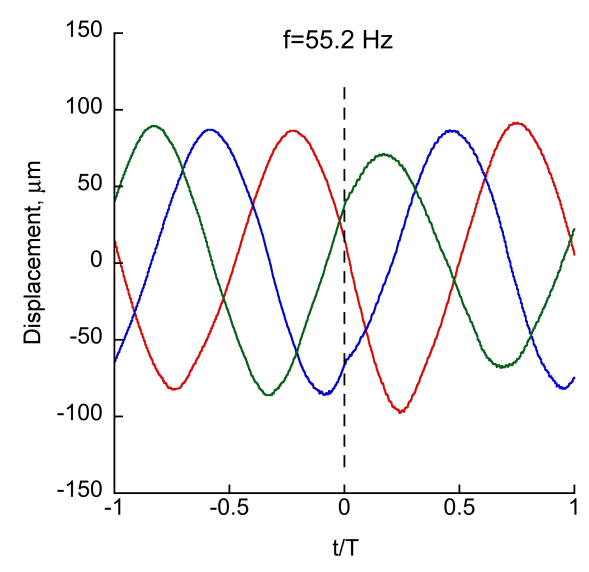

(b) Sting displacement under $I=0.015 \mathrm{~N}$-s impact with vibration motor set at $f=55.2 \mathrm{~Hz}$

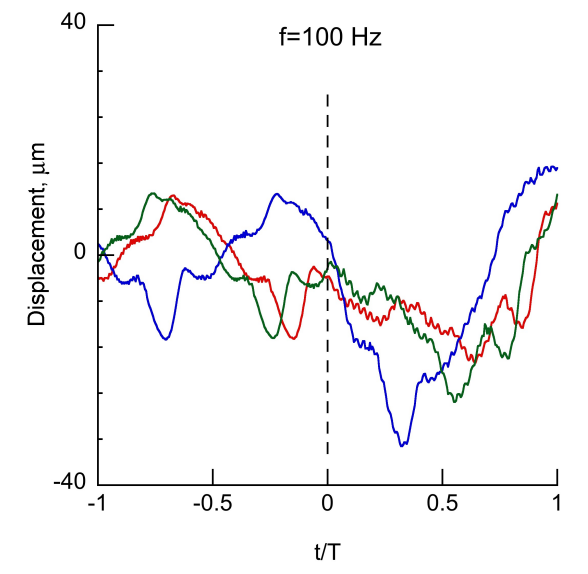

(c) Sting displacement under $I=0.015 \mathrm{~N}$-s impact with vibration motor set at $f=100 \mathrm{~Hz}$

Figure 5. Sting displacement. Colors represent three different random trigger phases.

$$
\omega_{n}=\frac{\omega_{d}}{\sqrt{\left(1-\zeta^{2}\right)}}
$$

where $x(t)$ is the sting displacement, $I$ is the instantaneous impulse, $m$ the floating total mass, $\omega_{d}$, the damped natural frequency, $\omega_{n}$, the undamped natural frequency, and $\zeta$, the damping coefficient ( $\phi$ is arbitrary and can be set to zero for this exercise). If Eq. 1 is differentiated to obtain velocity and evaluated at $t=0$, the impulse takes the particularly simple form of

$$
I=v_{0} m
$$

with $v_{0}$ the slope of the displacement response curve at $t=0$. The impulse can also be obtained by considering the full expression (Eq. 1) and least-square fitting a damped sine wave to the experimental response data. 
Each of these methods are evaluated in two examples shown in Figs. 6(a) and 6(b) to predict the magnitude of the impulse and also showing the respective accuracy. The error from the curve-fit method was less than $1 \%$ deviation from the calculated fully elastic impulse (impact) value, whereas the slope method yielded an error under $3 \%$. Before concluding which method is preferable, however, the analysis must be extended to the case of an initially vibrating target with both simple and complex vibration waveforms.

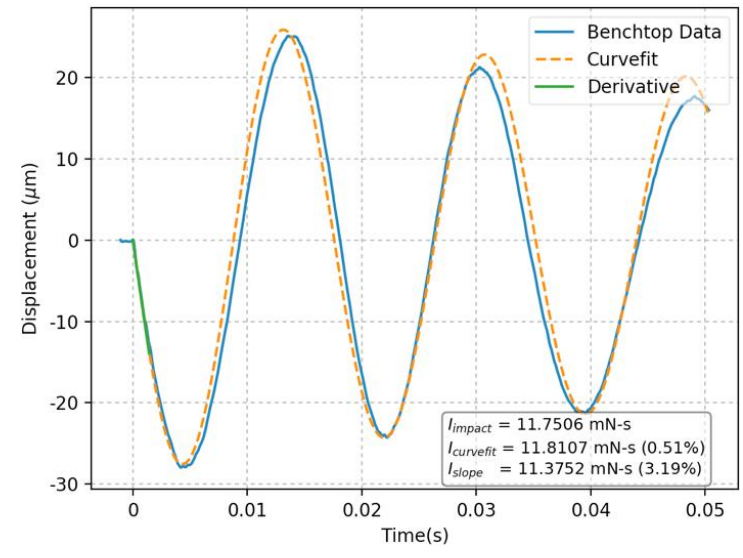

(a) Experiment 1

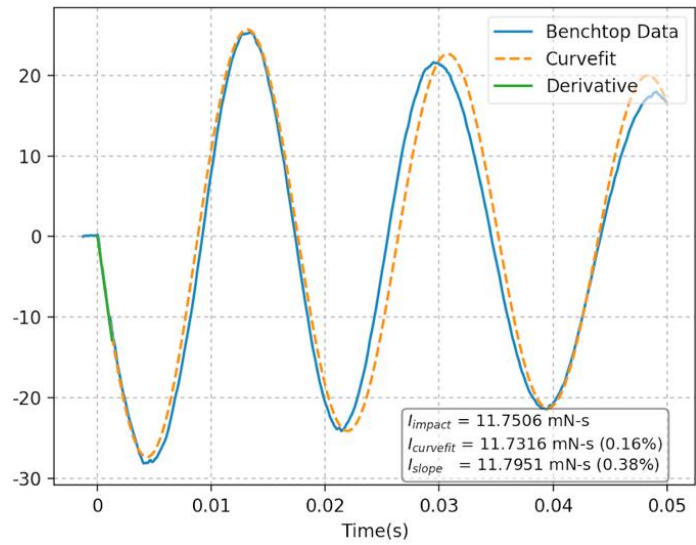

(b) Experiment 2

Figure 6. Example of determination of impulse from displacement data using initial slope and curve fit methods

\section{Description of Computations}

The governing equations are the nonequilibrium compressible Navier-Stokes equations using the Park I thermochemistry model ${ }^{15}$ and the Landau-Teller translational-vibrational energy exchange model. ${ }^{16}$ The complete description of the governing equations is presented in Kianvashrad and Knight. ${ }^{17}$ The governing equations are solved using a $\mathrm{C}^{++}$code developed by the authors, which implements Message Passing Interface (MPI). The code is a finite volume method using the Roe flux scheme and MUSCL (Monotone Upstream Scheme for Conservation Laws) reconstruction based upon the primitive variables. Viscous fluxes are calculated using a central difference method. A Data Parallel Line Relaxation (DPLR) method is used for time integration to achieve a high CFL (Courant-Friedrichs-Lewy) number. The simulations were performed using a 48 core Linux cluster.

The schematic of the computational domain for the laser discharge in front of the hemisphere cylinder is shown in Fig. 7. The boundary conditions are: streamwise axis from point A to B, isothermal no-slip wall from $\mathrm{B}$ to $\mathrm{D}$, zero gradient from $\mathrm{D}$ to $\mathrm{E}$, and fixed boundary condition from $\mathrm{E}$ to $\mathrm{F}$ and from $\mathrm{F}$ to $\mathrm{A}$. The computational domain is divided into multiple zones using a structured grid with uniform spacing along the surface and normal to the wall. Table 1 shows the grid properties of computations.

\section{Results}

\section{A. Efficiency of the Laser-Induced Breakdown Process}

To determine the efficiency of the laser-induced breakdown process, the energy transmitted through the breakdown region $\left(E_{t}\right)$ was compared to the energy input into the breakdown region $\left(E_{0}\right)$ for a focusing system consisting of a single 400-mm focal-length lens. The energy input into the breakdown region was varied by changing the energy at the laser head. The threshold energy $\left(E_{t h}\right)$ is the laser energy input to the breakdown region when breakdown first started to occur at a given pressure. Fig. 8 shows that prior to reaching the threshold energy, the energy transmitted through the breakdown region is equivalent to the energy input to the breakdown region $\left(E_{t} / E_{0} \approx 1\right)$. Once the energy input to the breakdown region is 


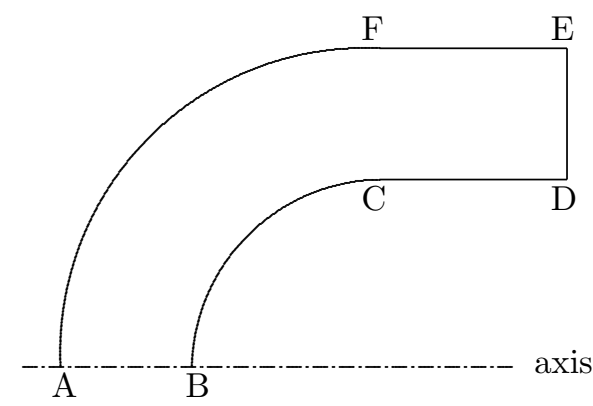

Figure 7. Computational domain.

Table 1. Computational grid (2.16 M cells).

\begin{tabular}{|c|c|c|c|c|c|}
\hline$i l$ & $j l$ & $k l$ & $\Delta \xi(\mu \mathrm{m})$ & $\Delta \eta(\mu \mathrm{m})$ & $\Delta \phi($ in degree $)$ \\
\hline 600 & 600 & 6 & 133.00 & 84.67 & 6 \\
\hline \multicolumn{6}{|c|}{ LEGEND } \\
\hline$i l$ & \multicolumn{5}{|c|}{ No. of points along surface } \\
\hline$j l$ & \multicolumn{5}{|c|}{ No. of points away from surface } \\
\hline$k l$ & \multicolumn{5}{|c|}{ No. of points in axisymmetric direction } \\
\hline$\xi$ & \multicolumn{5}{|c|}{ Direction along surface } \\
\hline$\eta$ & \multicolumn{5}{|c|}{ Direction away from surface } \\
\hline$\phi$ & \multicolumn{5}{|c|}{ Rotational direction about axis } \\
\hline
\end{tabular}

equivalent to the threshold energy $\left(E_{0} / E_{t h}=1\right)$, then breakdown begins to occur. The amount of energy lost through the breakdown region $\left(\Delta E=E_{0}-E_{t}\right)$ relative to the input pulse energy appears to reach a maximum at approximately $30-40 \%$ of the input pulse energy for the pressures tested with this focusing setup.

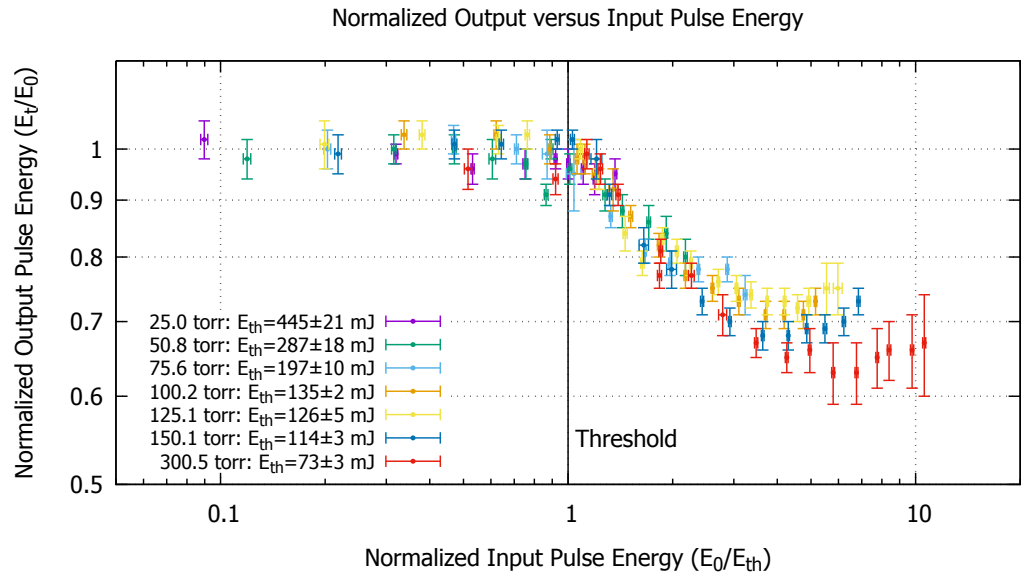

Figure 8. Pulse energy transmitted through the breakdown region as a function of input pulse energy, normalized by threshold energy for breakdown. Error bars are standard deviation for 300 samples.

Additionally, we examined the effects of several focusing setups to determine if there would be any efficiency gains for energy deposition. Three focusing setups were tested at standard atmospheric conditions ( $T=300 \mathrm{~K}, P=767$ torr). We compared the efficiencies for our unexpanded ( $\approx 7 \mathrm{~mm}$ diameter) beam focused by a $400 \mathrm{~mm}$ lens; a two times expanded beam, produced with $-200 \mathrm{~mm}$ and $400 \mathrm{~mm}$ lenses in a 
Galilean telescope configuration, focused by a $400 \mathrm{~mm}$ lens; and the unexpanded beam focused by $50 \mathrm{~mm}$ lens, which is comparable to the lenses used in other works. We observed a marked improvement in the efficiency of energy absorption ( $>90 \%$ at high energy) when the focal volume was reduced in the latter two cases (Fig. 9); however, short focal lengths are not possible for this application because of the facility dimensions. Nonetheless, beam expansion could be used to improve efficiency.

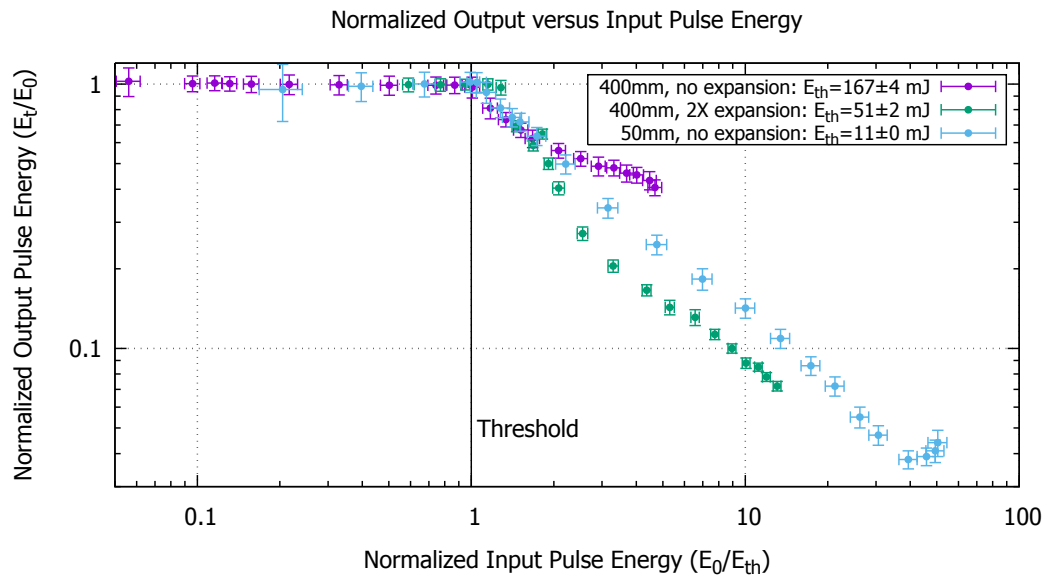

Figure 9. Fractional energy transmission versus normalized input energy, measured relative to threshold for the various focusing configurations at standard atmospheric conditions.

\section{B. Laser Discharge in Quiescent Air}

Several schlieren data sets were taken of the shock and thermal perturbation resulting from the laser-induced breakdown process. For the figures shown in this section, only a single 400-mm focal-length lens was used to induce breakdown of the air at vacuum. These data were taken for pressures ranging from 70-762 torr and laser energies ranging from $170-650 \mathrm{~mJ}$ per pulse.

The evolution of the disturbance created by the laser-induced breakdown at $p=150$ torr and $T=300 \mathrm{~K}$ is shown in Fig. 10. The energy at the laser head is $350 \mathrm{~mJ}$ and the energy absorbed by the gas is $\Delta E=$ $61 \pm 9 \mathrm{~mJ}$. The schlieren images are an average of 100 frames, each taken at the time $t$ indicated after a laser pulse was fired. Fig. 10(a) shows that the initial shock wave has a slightly elliptical shape. After approximately $25 \mu$ s the shape becomes circular. At approximately $t=90 \mu \mathrm{s}$ (Fig. 10(p)) a toroidal vortex forms and a jet begins to emerge from the center of the torus (Glumac et al ${ }^{18}$ ). In Figs. 10(q) to 10(u) the growth of the laser-generated vortical flow toward the laser head is visible.

As the laser energy increases, the shock wave that initially emanates from the thermal core becomes less spherical. The location of the center of the thermal core also appears to move closer to the incoming laser beam. At near-threshold conditions for $p=150.5$ torr, the thermal core appears to be centered closer to the expected focus of the focusing optics. Increasing the energy at the laser head by 3.8 times causes the center of the thermal core to shift approximately $14 \mathrm{~mm}$ toward the incoming laser beam. We also observe that this translation is reduced when beam expansion or a shorter focal length lens are used. Increasing the energy at the laser head for the case of the twice expanded beam focused by the $400 \mathrm{~mm}$ lens from threshold to 9.3 times the threshold intensity causes the center of the thermal core to shift by only about $8 \mathrm{~mm}$. The translation of the thermal core is shown in Fig. 11 for both cases.

Fig. 12 compares the computational and experimental shock radius versus time for an ambient pressure of $p=150$ torr and temperature $T=300 \mathrm{~K}$ in air. The laser discharge energy is $350 \mathrm{~mJ}$ and the measured energy absorbed by the gas is $\Delta E=61 \pm 9 \mathrm{~mJ}$. Data from the schlieren images were analyzed using Matlab image processing tools. In each image, there are two sides for the shock - the bright side and the dark side. Both of these radii are indicated in the figure. In the numerical simulation, different initial conditions were considered for a fixed energy deposition of $61 \mathrm{~mJ}$. The initial density of the discharge is equal to 


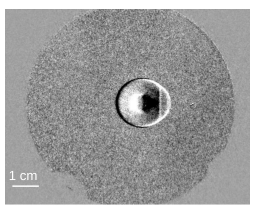

(a) $\mathrm{t}=15 \mu \mathrm{s}$

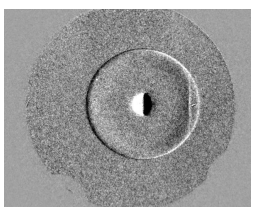

(g) $\mathrm{t}=45 \mu \mathrm{s}$

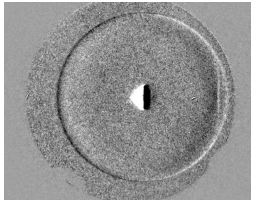

(m) $\mathrm{t}=75 \mu \mathrm{s}$

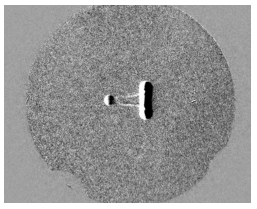

(s) $\mathrm{t}=400 \mu \mathrm{s}$

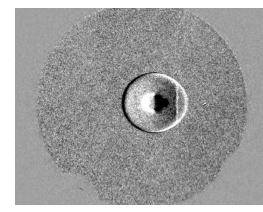

(b) $\mathrm{t}=20 \mu \mathrm{s}$

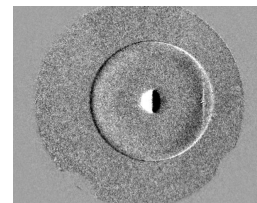

(h) $\mathrm{t}=50 \mu \mathrm{s}$

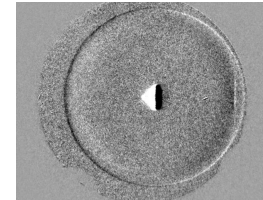

(n) $\mathrm{t}=80 \mu \mathrm{s}$

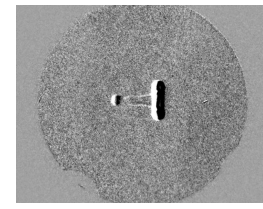

(t) $\mathrm{t}=500 \mu \mathrm{s}$

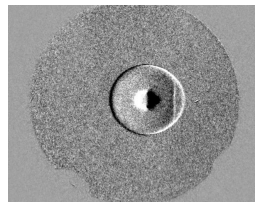

(c) $\mathrm{t}=25 \mu \mathrm{s}$

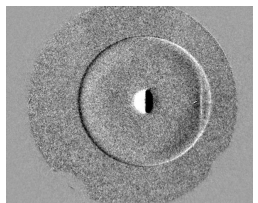

(i) $\mathrm{t}=55 \mu \mathrm{s}$

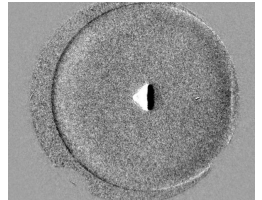

(o) $\mathrm{t}=85 \mu \mathrm{s}$

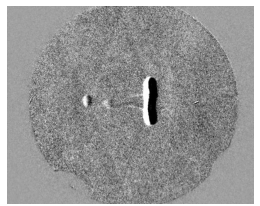

(u) $\mathrm{t}=1000 \mu \mathrm{s}$

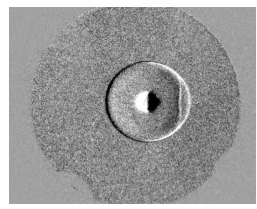

(d) $\mathrm{t}=30 \mu \mathrm{s}$

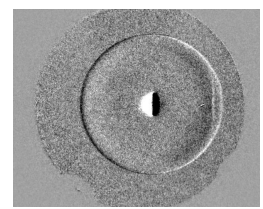

(j) $\mathrm{t}=60 \mu \mathrm{s}$

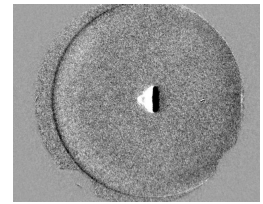

(p) $\mathrm{t}=90 \mu \mathrm{s}$

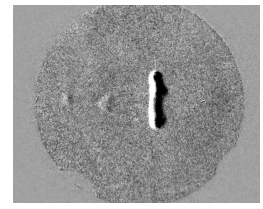

(v) $\mathrm{t}=2000 \mu \mathrm{s}$

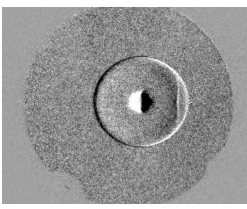

(e) $\mathrm{t}=35 \mu \mathrm{s}$

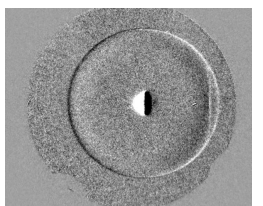

(k) $\mathrm{t}=65 \mu \mathrm{s}$

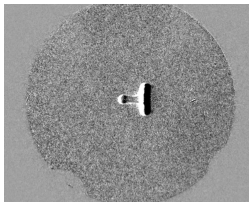

(q) $\mathrm{t}=200 \mu \mathrm{s}$

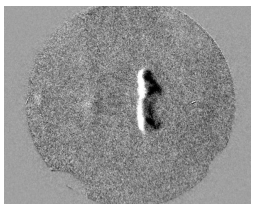

(w) $\mathrm{t}=3000 \mu \mathrm{s}$

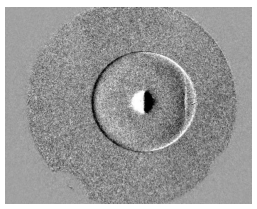

(f) $\mathrm{t}=40 \mu \mathrm{s}$

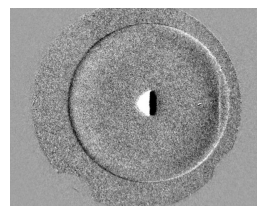

(l) $\mathrm{t}=70 \mu \mathrm{s}$

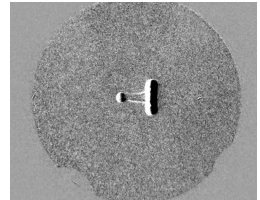

(r) $\mathrm{t}=300 \mu \mathrm{s}$

Figure 10. Schlieren Images for $p=150$ torr and $\Delta E=61 \mathrm{~mJ}$.

the freestream density. The initial radius of the laser discharge (and hence the initial temperature of the discharge) was varied. Also, for the same initial radius of $3 \mathrm{~mm}$, two different conditions were considered for partitioning the energy between the translational-rotational temperature and the vibrational temperature. In the first case, the translational-rotational and the vibrational temperatures are equal. In the second case, all the energy deposited in the gas by the laser goes to translational-rotational energy; therefore, the vibrational temperature is the ambient temperature. It is evident that the initial radius of laser discharge sphere and also the different types of energy partitioning do not have any significant effect on the shock radius versus time except in the very early stage (less than $10 \mu \mathrm{s}$ ) after the discharge. After approximately $10 \mu$ s the shock speed has reduced to within a few percent of the acoustic speed as shown in Fig. 13. This figure also shows that the blast wave theory is not valid for these experiments since the shock Mach number rapidly reduces to one. The experimental Mach number is calculated point to point while the computational results were averaging over 50 to 100 data points.

\section{Laser Discharge in Front of a Hemisphere Cylinder at Mach 2.0}

A computation was performed at the freestream condition of Table 2 where the Reynolds number $R e_{\infty}$ is based on the hemisphere radius. The isothermal wall temperature is $295.49 \mathrm{~K}$. The initial condition 


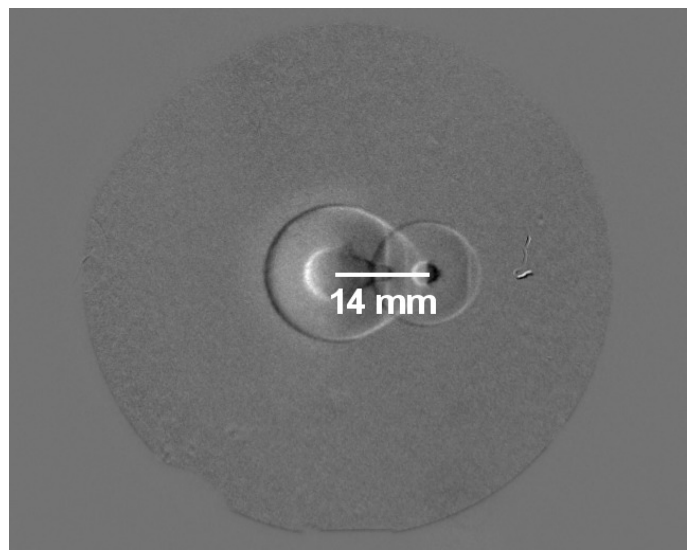

(a) Unexpanded beam focused by $400 \mathrm{~mm}$ lens with absorbed energies of $5 \mathrm{~mJ}$ and $156 \mathrm{~mJ}$ at $15 \mu$ s delay

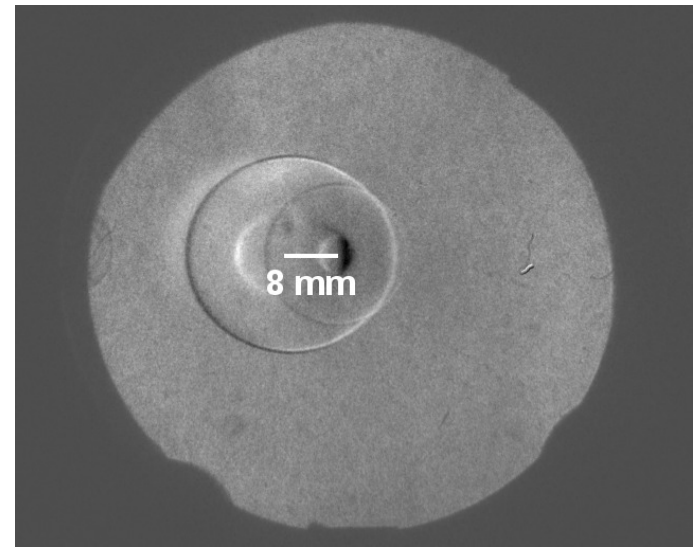

(b) Two times expanded beam focused by $400 \mathrm{~mm}$ lens with absorbed energies of $30 \mathrm{~mJ}$ and $430 \mathrm{~mJ}$ at $20 \mu$ s delay

Figure 11. Translation of the thermal core between threshold and the maximum applied laser energy.

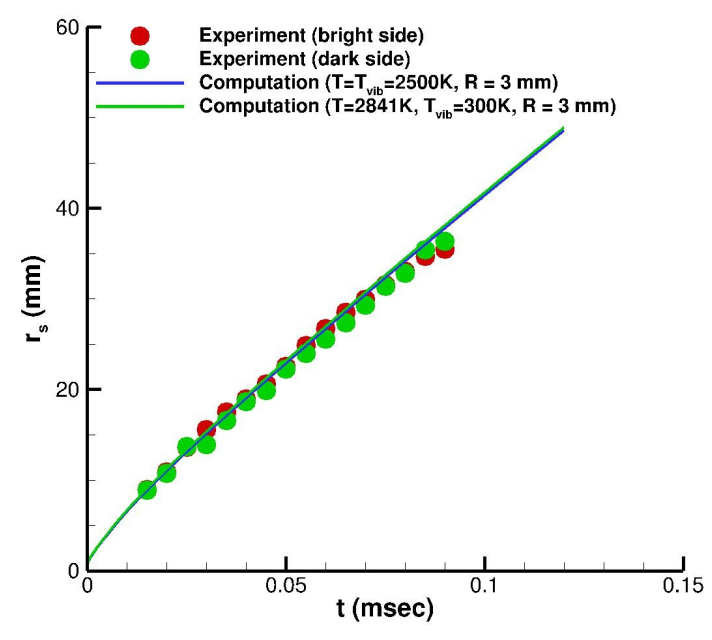

(a) Effect of energy distribution

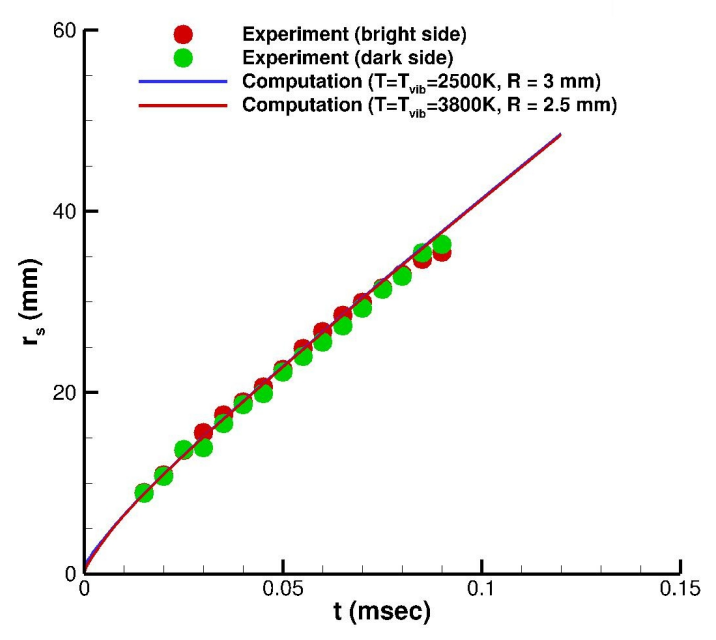

(b) Effect of initial impulse radius

Figure 12. Comparison of shock radius versus time of experimental and numerical results (uncertainty of radius capturing between the two side is less than $3 \%$ ).

is the converged solution for the hemisphere cylinder in the Mach 2.0 flow. The absorbed energy of the laser discharge by the gas is $50 \mathrm{~mJ}$. The laser discharge of $50 \mathrm{~mJ}$ is assumed to increase the translationalrotational and vibrational temperature equally with a resultant value of $2238 \mathrm{~K}$ for the initial discharge radius of $2.54 \mathrm{~mm}$. The initial density of the discharge is equal to the freestream density. The discharge location is 0.83 hemisphere radii upstream of the hemisphere tip. The flow is from left to right which is considered as the $x$-direction.

Figs. 14 and 15 depict contour plots of Mach number (top section of each figure) and vibrational temperature of $\mathrm{N}_{2}$ (bottom section of each figure) at specified times. Figs. 16 and 17 show the contour plots of density gradient magnitude (top section) and pressure divided by freestream pressure (bottom section). At 


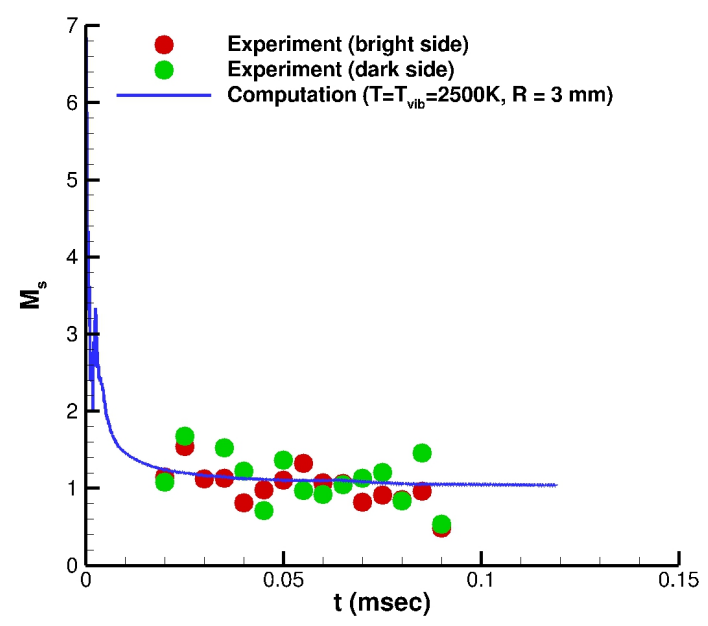

Figure 13. Shock Mach number versus time of experimental and numerical results.

Table 2. Freestream conditions.

\begin{tabular}{c|c} 
Variables & Freestream condition \\
\hline$M_{\infty}$ & 2.0 \\
$T_{\infty}$ & $172.74 \mathrm{~K}$ \\
$P_{\infty}$ & $17.623 \mathrm{kPa}$ \\
$\rho_{\infty}$ & $3.542 \mathrm{E}-01 \mathrm{~kg} / \mathrm{m}^{3}$ \\
$Y_{\mathrm{N}_{2}}$ & 0.765 \\
$Y_{\mathrm{O}_{2}}$ & 0.235 \\
$R e_{\infty}$ & $4.09982 \mathrm{E}+05$
\end{tabular}

$t=0.48 \mu$ s (Figs. 14(a) and 16(a)), the heated region is visible. The blast wave is so close to the heated region that it is unrecognizable. At $t=13.42 \mu \mathrm{s}$ (Figs. 14(b) and 16(b)), the blast wave is already interacting with the bow shock. Based on the Yan et al. ${ }^{19}$ study, the expansion of the heated region occurs as a result of the radial velocity at the edge of the heated region. At the same time, the vibrational temperature of $\mathrm{N}_{2}$ remains almost the same value since the calculation omits translational-vibrational energy exchange. At $t=28.76 \mu$ s (Figs. 14(c) and 16(c)), the transmitted shock - due to blast wave and bow shock interaction - reaches the hemisphere surface. Moreover, the heated region has begun to interact with the bow shock. This interaction compresses the heated region. As a result of the lower Mach number inside the heated region, the bow shock moves forward (the "lensing" phenomenon observed previously in other's studies such as Adelgren et al. ${ }^{5}$ and Schülein et al. ${ }^{7}$ ). The interaction of the distorted bow shock and the original bow shock outside the heated region forms a $\lambda$-shock as reported by Schülein et al. ${ }^{7}$ Figs. 14(d)-(f) show the formation of vortex rings as a consequence of the Richtmyer-Meshkov instability, which is caused by the difference in density gradient and is observable in Figs. 16(d)-(f). It should be noted that the shock formed due to laser discharge completely passed the bow shock at Figs. 14(f) and 16(f). Figs. 15 and 17 demonstrate the propagation of the vortices along the hemisphere surface. Additionally, as the heated region moves inside the bow shock, the convex part of the bow shock moves backward and in the last two figures (Figs. 15(f) and $17(\mathrm{f}))$ the bow shock reaches its original position.

Fig. 18 shows the change of force in $x$ direction due to pressure (pressure drag force) nondimensionalized by $F_{x}^{o}$, which is the undisturbed force in $x$ direction, calculated for the entire hemisphere cylinder versus time. The dimensionless time $t U_{\infty} / R$ is the lower horizontal axis where $R$ is the hemisphere radius while the dimensional time is the upper axis. Time is measured from the instantaneous laser discharge. The dashed 


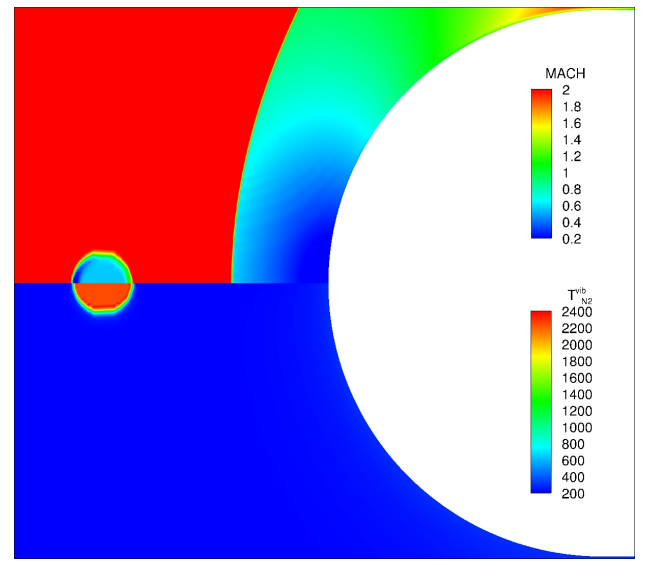

(a) $\mathrm{t}=0.48 \mu \mathrm{s}$

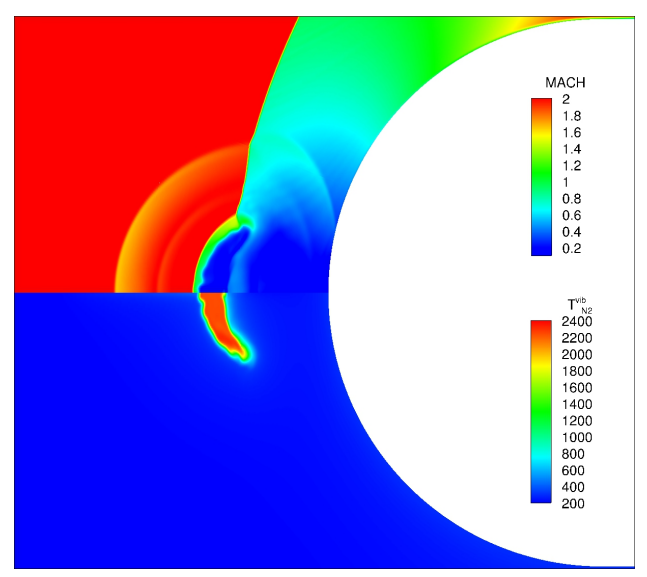

(c) $\mathrm{t}=28.76 \mu \mathrm{s}$

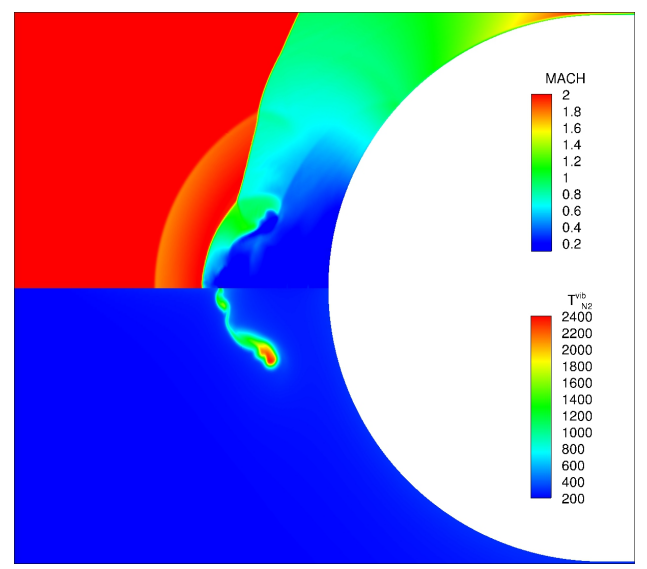

(e) $\mathrm{t}=45.07 \mu \mathrm{s}$

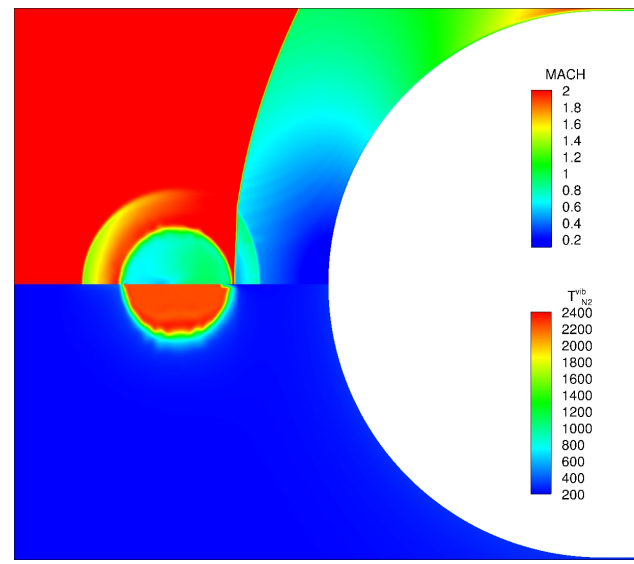

(b) $\mathrm{t}=13.42 \mu \mathrm{s}$

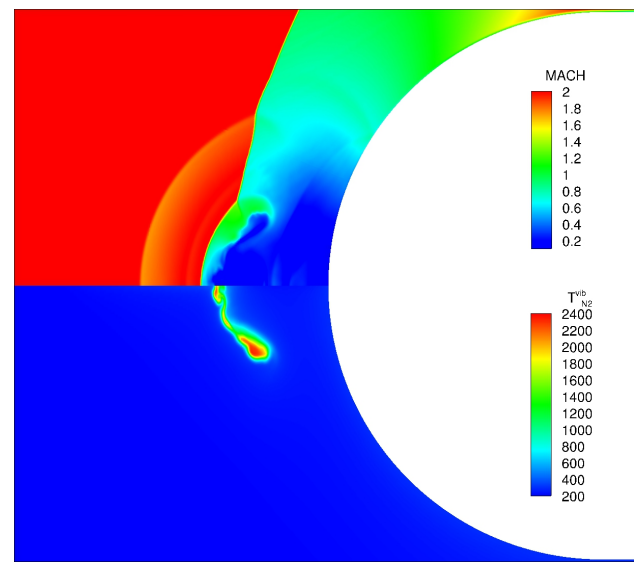

(d) $\mathrm{t}=36.92 \mu \mathrm{s}$

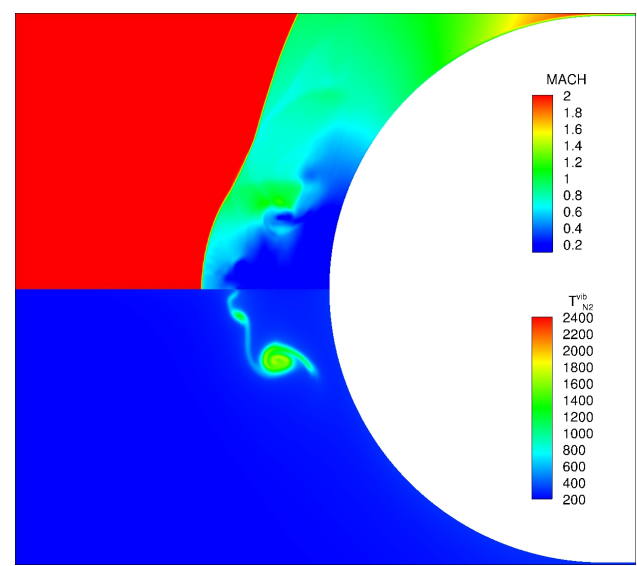

(f) $\mathrm{t}=66.63 \mu \mathrm{s}$

Figure 14. Contour plots of Mach number and vibrational temperature of $\mathbf{N}_{2}$ for $\Delta E=50 \mathrm{~mJ}$. 


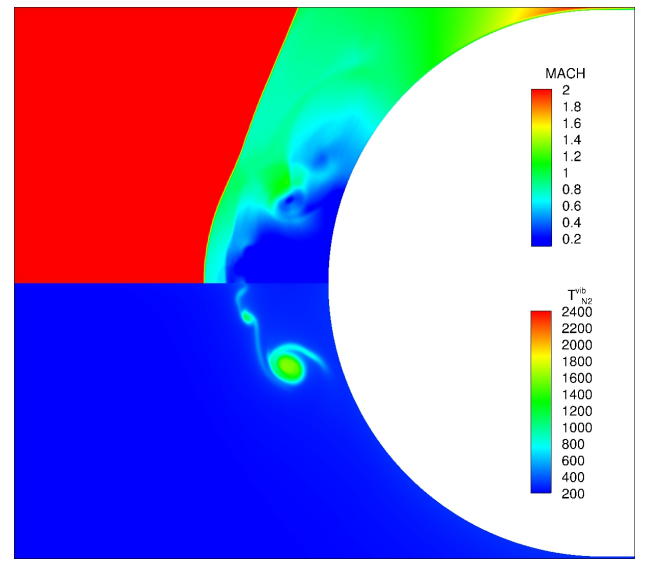

(a) $\mathrm{t}=78.15 \mu \mathrm{s}$

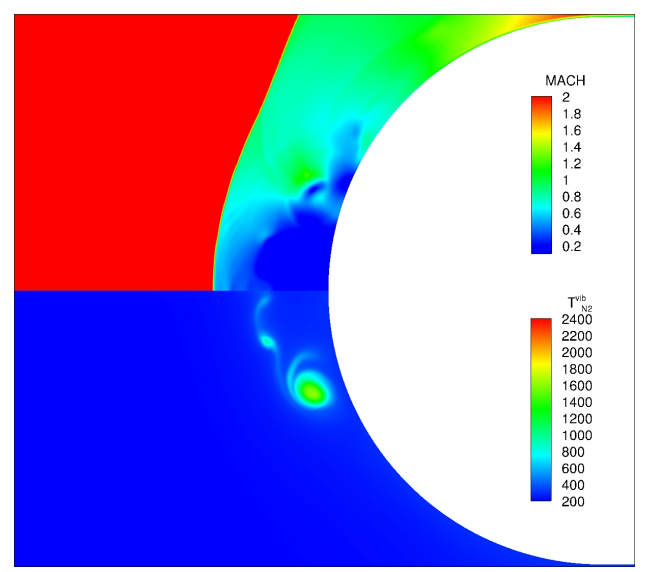

(c) $\mathrm{t}=104.03 \mu \mathrm{s}$

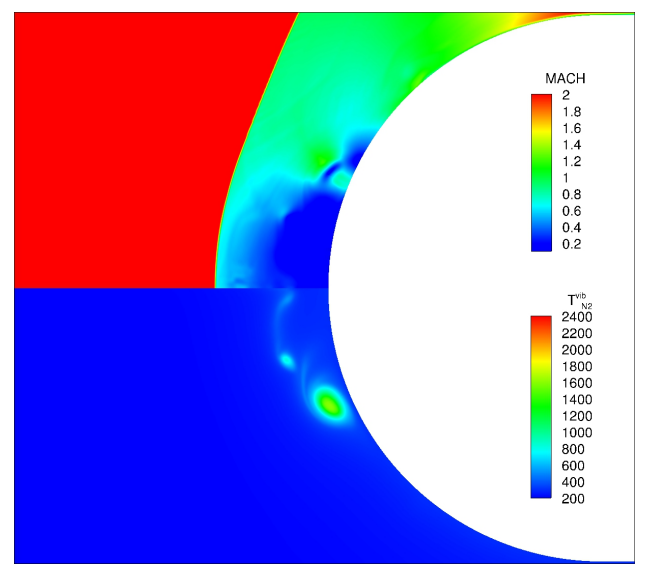

(e) $\mathrm{t}=129.92 \mu \mathrm{s}$

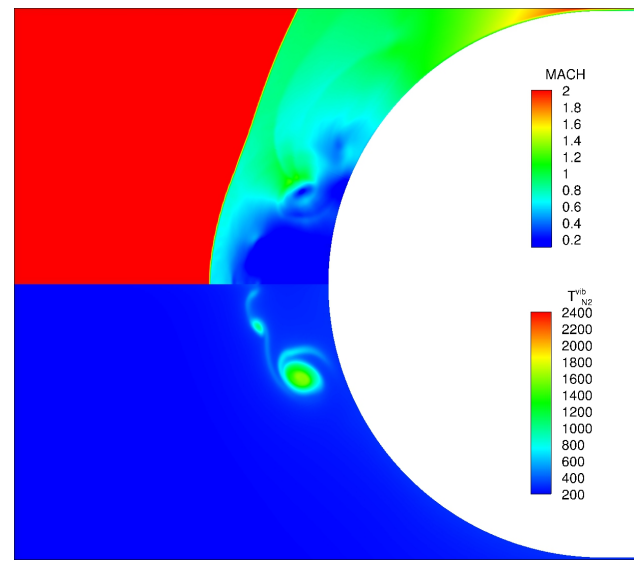

(b) $\mathrm{t}=91.09 \mu \mathrm{s}$

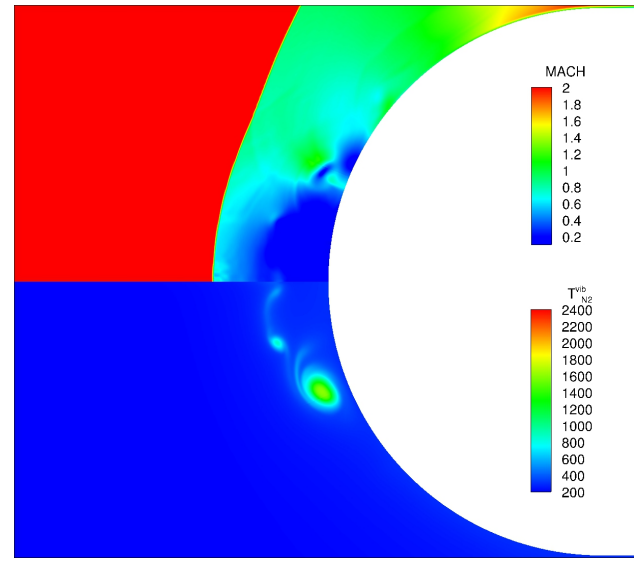

(d) $\mathrm{t}=116.98 \mu \mathrm{s}$

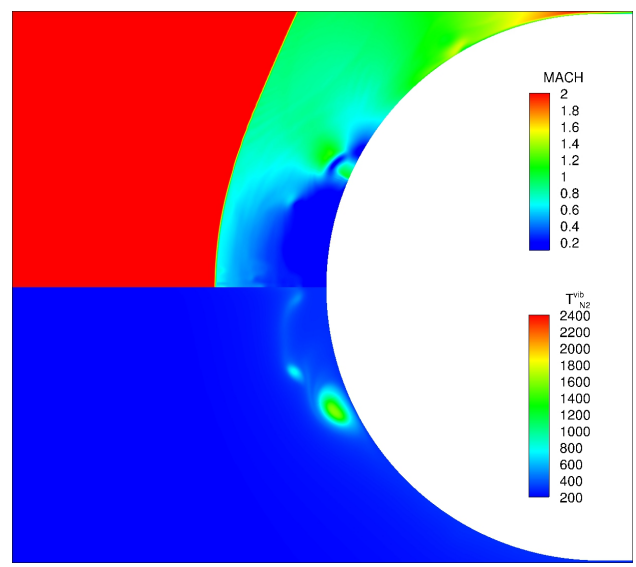

(f) $\mathrm{t}=142.87 \mu \mathrm{s}$

Figure 15. Contour plots of Mach number and vibrational temperature of $\mathbf{N}_{2}$ for $\Delta E=50 \mathrm{~mJ}$. 


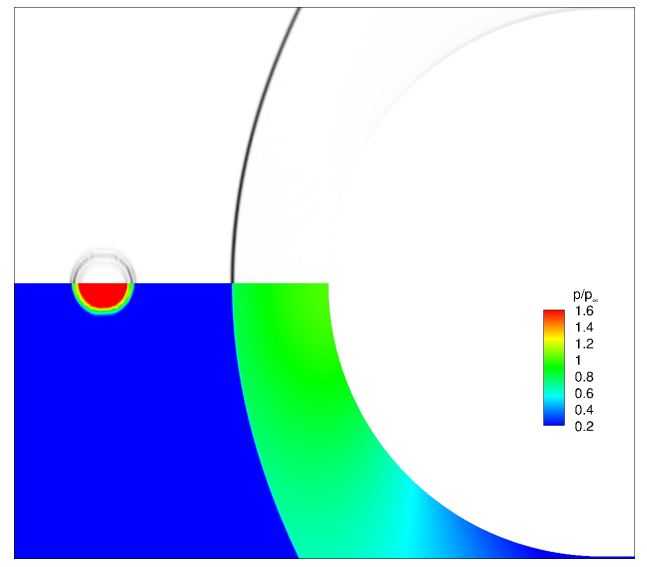

(a) $\mathrm{t}=0.48 \mu \mathrm{s}$

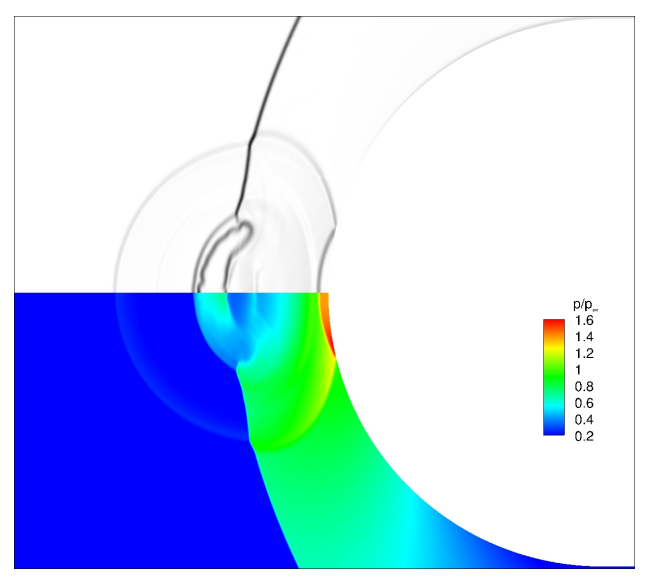

(c) $\mathrm{t}=28.76 \mu \mathrm{s}$

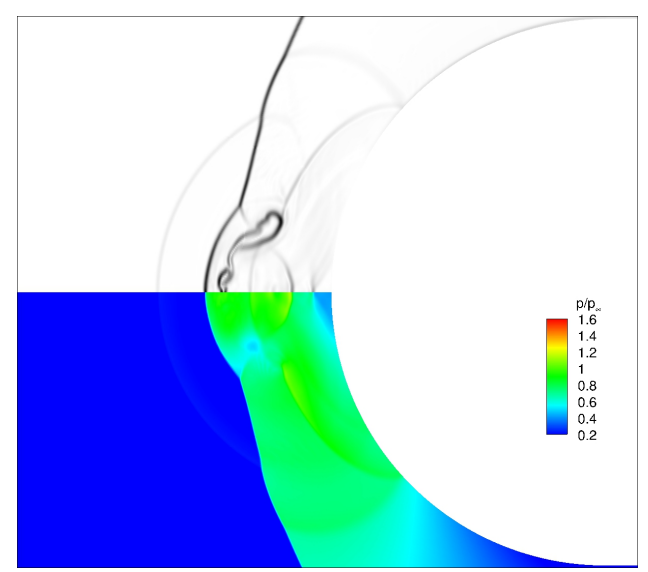

(e) $\mathrm{t}=45.07 \mu \mathrm{s}$

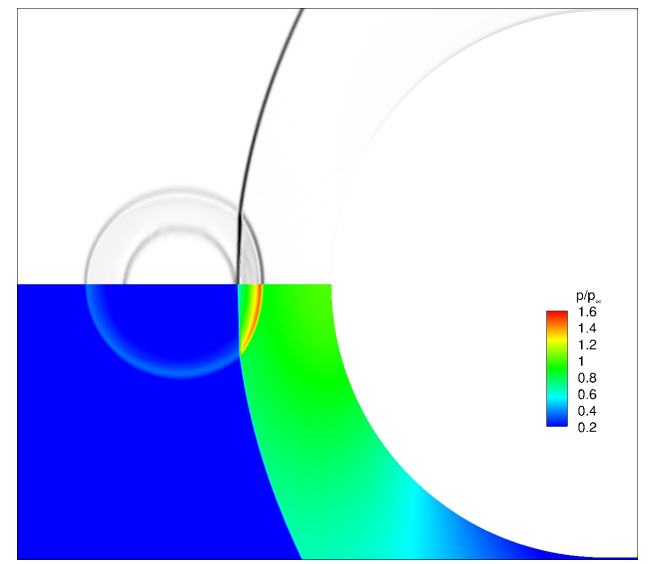

(b) $\mathrm{t}=13.42 \mu \mathrm{s}$

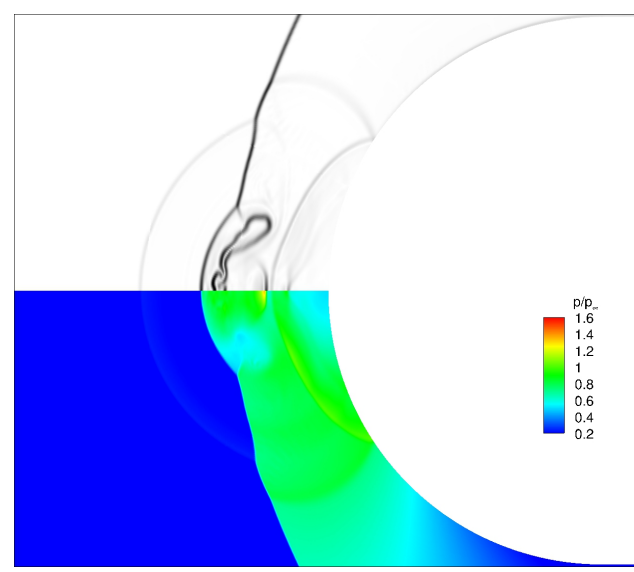

(d) $\mathrm{t}=36.92 \mu \mathrm{s}$

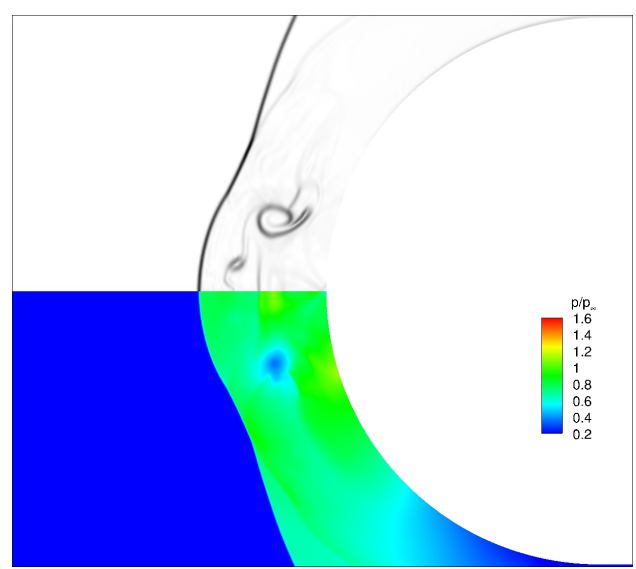

(f) $\mathrm{t}=66.63 \mu \mathrm{s}$

Figure 16. Contour plots of density gradient and dimensionless pressure for $\Delta E=50 \mathrm{~mJ}$. 


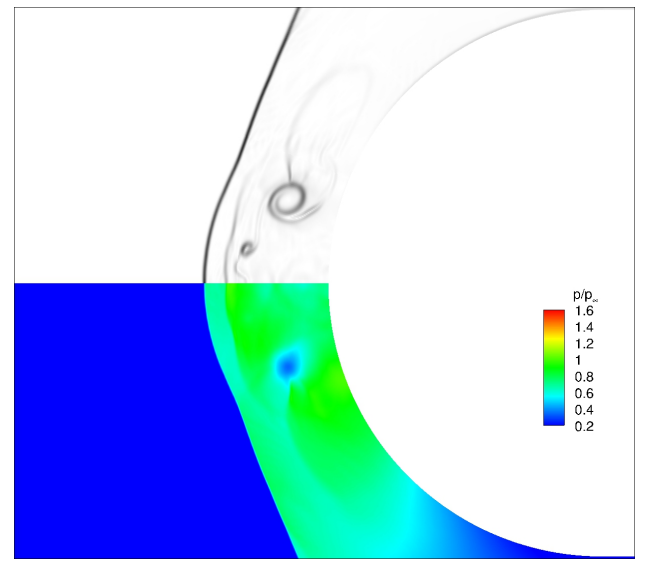

(a) $\mathrm{t}=78.15 \mu \mathrm{s}$

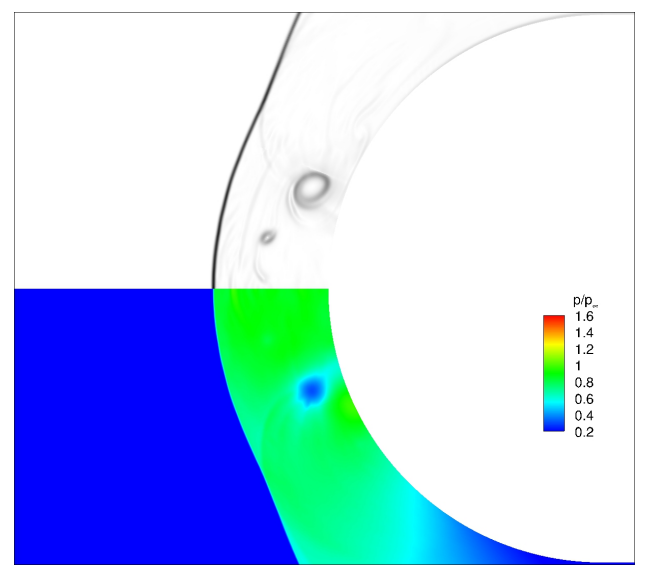

(c) $\mathrm{t}=104.03 \mu \mathrm{s}$

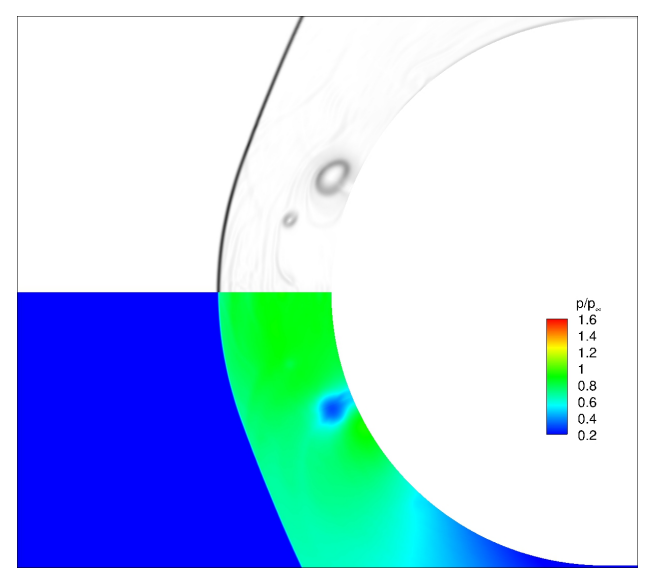

(e) $\mathrm{t}=129.92 \mu \mathrm{s}$

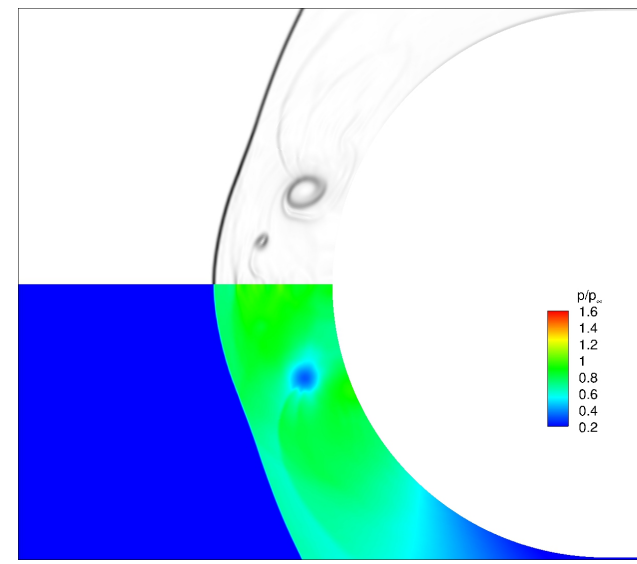

(b) $\mathrm{t}=91.09 \mu \mathrm{s}$

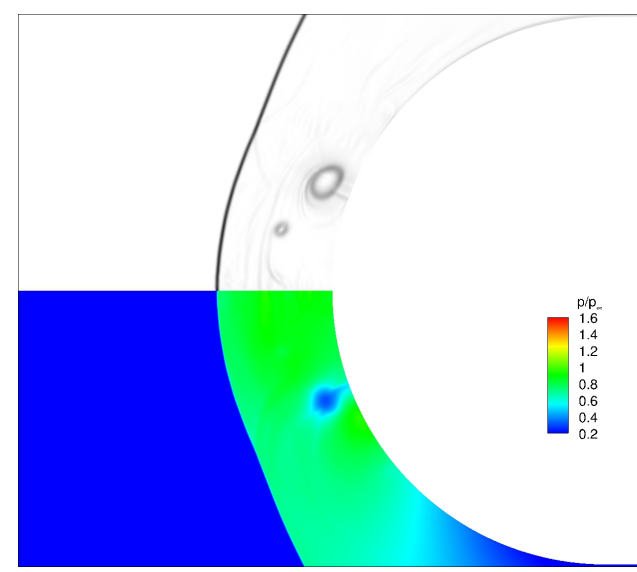

(d) $\mathrm{t}=116.98 \mu \mathrm{s}$

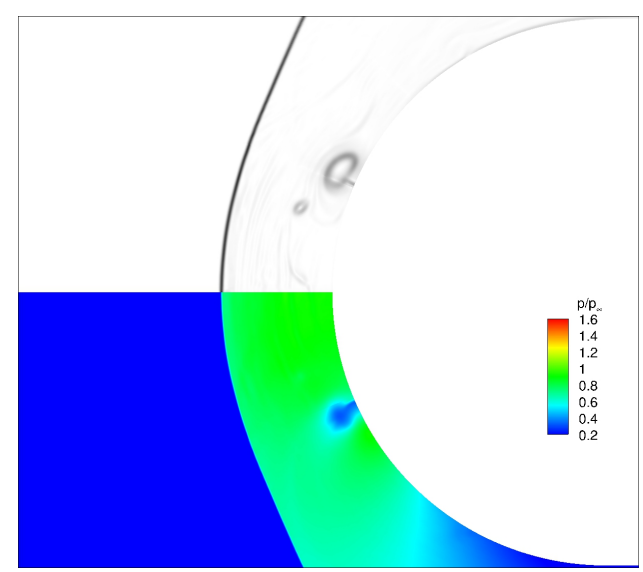

(f) $\mathrm{t}=142.87 \mu \mathrm{s}$

Figure 17. Contour plots of density gradient and dimensionless pressure for $\Delta E=50 \mathrm{~mJ}$. 
line shows $\Delta F_{x}$ equal to zero. Deviation of the change in force from zero line denoted by the dashed line shows the long lasting effect of the laser discharge over the pressure drag force.

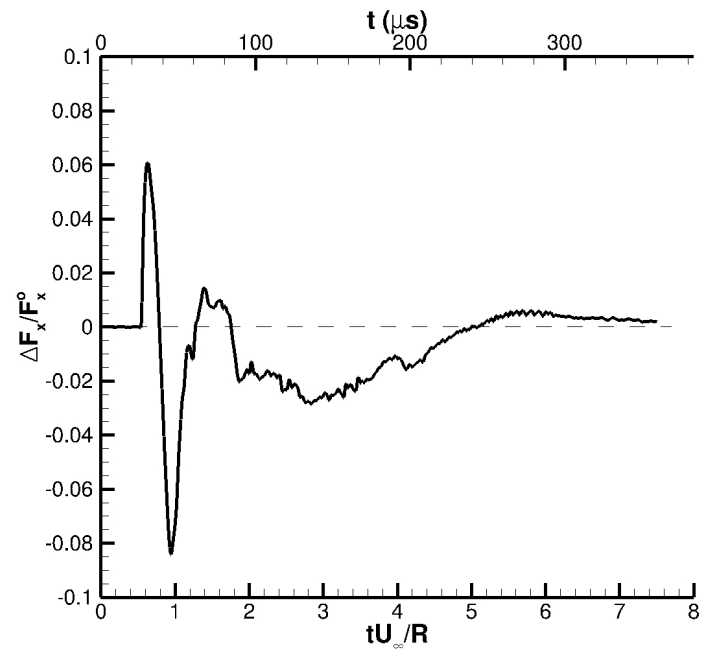

Figure 18. Dimensionless force change versus time (pressure drag only).

The first peak in Fig. $18(t \approx 28.76 \mu$ s related to Figs. $14(\mathrm{c})$ and $16(\mathrm{c}))$ is related to the shock wave interaction with the hemisphere tip, which increases the pressure and hence the pressure drag force. The expansion wave transmitted from the interaction of the heated region and bow shock reduces the pressure, which reaches a negative peak at about $t \approx 45.07 \mu$ s after discharge (Figs. 14(e) and 16(e)). A compression wave creates the second peak at about $66.63 \mu \mathrm{s}$ (Figs. 14(f) and 16(f)). The presence of the vortices about the hemisphere surface, which are visible in the vibrational temperature of $\mathrm{N}_{2}$ in Figs. 14 and 15, create the pressure reduction, as seen in Figs. 16 and 17 and maintain a drag reduction up to $t=250 \mu \mathrm{s}$.

The gas dynamic energetic efficiency is defined as the ratio of energy saved due to the interaction of the laser discharge to the energy absorbed by the gas. The energy saved is the negative of the change in the impulse times the inflow velocity. Thus, the gas dynamic energetic efficiency is calculated as

$$
\text { GDEE }=-\frac{u_{\infty} \Delta \text { Impulse }}{\Delta E}
$$

The change in the impulse due to laser discharge is calculated using Fig. 18. The gas dynamic energetic efficiency of this simulation is $3.22(322 \%)$.

\section{Future Work}

The wind tunnel experiments for interaction of the off-body laser discharge with the hemisphere cylinder remain to be performed. The experimental data will be analyzed to determine the gas dynamic efficiency (i.e., the energy saved by drag reduction over the interval of the interaction divided by the energy absorbed by the gas). The simulations will be compared with the experiment to assess the capability for prediction of the drag reduction given the measured energy absorbed by the gas.

\section{Conclusion}

The paper presents a combined computational and experimental study of the interaction of an offbody laser discharge with a hemisphere cylinder in supersonic flow. The objectives are 1) experimental determination of the drag reduction and energetic efficiency of the laser discharge, and 2) assessment of the capability for accurate simulation of the interaction. In the first phase, laser discharge in quiescent air at pressures from 150 torr to 762 torr and temperature of $300 \mathrm{~K}$ is examined. Experimental measurements of 
the actual energy absorbed by the gas are performed. Simulations of the shock wave radius versus time are in close agreement with schlieren imaging. In the second phase, the interaction of a $50 \mathrm{~mJ}$ laser discharge with a hemisphere-cylinder is investigated computationally at Mach 2. The gas dynamic energetic efficiency of the laser discharge is 3.22 .

\section{References}

${ }^{1}$ Artem'ev, V., Bergel'son, V., Nemchinov, I., Orlova, T., Smirnov, A., and Khazins, V., "Change of Regime in Supersonic Flow Past on Obstacle Preceded by a Thin Channel of Reduced Density," Izvestiya Akademii Nauk SSSR, Mekhanika Zhidkostii Gaza, Vol. 5, No. September-October, 1989, pp. 146-151.

${ }^{2}$ Tretyakov, P., Garanin, A., Kraynev, V., Tupikin, A., and Yakovlev, V., "Investigation of Local Laser Energy Release Influence on Supersonic Flow by Methods of Aerophysical Experiments," International Conference on Methods of Aerophysical Research, Novosibirsk, Russia,, 1996.

${ }^{3}$ Riggins, D., Nelson, H., and Johnson, E., "Blunt-Body Wave Drag Reduction Using Focused Energy Deposition," AIAA Journal, Vol. 37, No. 4, 1999, pp. 460-467.

${ }^{4}$ Lashkov, V., Mashek, I., Anisimov, Y., Ivanov, V., Kolesnichenko, Y., Ryvkin, M., and Gorynya, A., "Gas Dynamic Effect of Microwave Discharge on Supersonic Cone-shaped Bodies," AIAA Paper 2004-0671, American Institute of Aeronautics and Astronautics, January 2004.

${ }^{5}$ Adelgren, R., Yan, H., Elliott, G., Knight, D., Beutner, T., and Zheltovodov, A., "Control of Edney IV Interaction by Pulsed Laser Energy Deposition," AIAA Journal, Vol. 43, No. 2, 2005, pp. 256-269.

${ }^{6}$ Knight, D., Kolesnichenko, Y., Brovkin, V., Khmara, D., Lashkov, V., and Mashek, I., "Interaction of MicrowaveGenerated Plasma with a Hemisphere Cylinder at Mach 2.1," AIAA Journal, Vol. 47, No. 12, 2009, pp. $2996-3010$.

${ }^{7}$ Schülein, E., Zheltovodov, A., Pimonov, E., and Loginov, M., "Experimental and Numerical Modeling of the Bow Shock Interaction with Pulse-Heated Air Bubbles," International Journal of Aerospace Innovations, Vol. 2, No. 3, 2010 , pp. 165-188.

${ }^{8}$ Kim, J., Matsuda, A., Sakai, T., and Sasoh, A., "Wave Drag Reduction with Acting Spike Induced by Laser-Pulse Energy Depositions," AIAA Journal, Vol. 49, No. 9, 2011, pp. 2076-2078.

${ }^{9}$ Kremeyer, K., Sebastian, K., and Shu, C., "Computational Study of Shock Mitigation and Drag Reduction by Pulsed Energy Lines," AIAA Journal, Vol. 44, No. 8, 2006, pp. 1720-1731.

${ }^{10}$ Plooster, M., "Shock Waves from Line Sources. Numerical Solutions and Experimental Measurements," The Physics of Fluids, Vol. 13, No. 11, 1970, pp. 2665-2675.

${ }^{11}$ Plooster, M., "Erratum: Shock Waves from Line Sources. Numerical Solutions and Experimental Measurements," The Physics of Fluids, Vol. 14, No. 10, 1971, pp. 2248-2248.

${ }^{12}$ Knight, D., "Survey of Aerodynamic Drag Reduction at High Speed by Energy Deposition," Journal of Propulsion and Power, Vol. 24, No. 6, November-December 2008, pp. 1153-1167.

${ }^{13}$ Dillon, J., Trimpi, R., and Schultz, A., "The NASA-Langley 20-Inch Supersonic Wind Tunnel," AIAA Paper 86-0765, American Institute of Aeronautics and Astronautics, March 1986.

${ }^{14}$ Giancoli, D., Physics for Scientists \& Engineers, Prentice Hall, Upper Saddle River, NJ, 3rd ed., 1965.

${ }^{15}$ Park, C., "On Convergence of Computation of Chemically Reacting Flow," AIAA Paper 1985-0247, American Institute of Aeronautics and Astronautics, January 1985.

${ }^{16}$ Landau, L. and Teller, E., "Zur Theorie der Schalldispersion," Physikalische Zeitschrift der Sowjetunion, Vol. 10, 1936, pp. 34-43.

${ }^{17}$ Kianvashrad, N. and Knight, D., "Simulation of Hypersonic Shock Wave Laminar Boundary Layer Interaction on Hollow Cylinder Flare," AIAA Paper 2016-0349, American Institute of Aeronautics and Astronautics, January 2016.

${ }^{18}$ Glumac, N., Elliott, G., and Boguszko, M., "Temporal and Spatial Evolution of a Laser Spark in Air," AIAA Journal, Vol. 43, No. 9, 2005, pp. 1984-1994.

${ }^{19}$ Yan, H., Adelgren, R., Boguszko, M., Elliott, G., and Knight, D., "Laser Energy Deposition in Quiescent Air," AIAA Journal, Vol. 41, No. 10, 2003, pp. 1988-1995. 